\title{
Machine learning classification of new asteroid families members
}

\author{
V. Carruba ${ }^{\oplus,}{ }^{1 \star}$ S. Aljbaae, ${ }^{2}$ R. C. Domingos, ${ }^{3}$ A. Lucchini ${ }^{\oplus 1}$ and P. Furlaneto ${ }^{1}$ \\ ${ }^{1}$ School of Natural Sciences and Engineering, São Paulo State University (UNESP), Guaratinguetá, SP 12516-410, Brazil \\ ${ }^{2}$ National Space Research Institute (INPE), Division of Space Mechanics and Control, C.P. 515, São José dos Campos, SP 12227-310, Brazil \\ ${ }^{3}$ São Paulo State University (UNESP), Sao João da Boa Vista, SP 13874-149, Brazil
}

Accepted 2020 May 21. Received 2020 May 19; in original form 2020 March 20

\begin{abstract}
Asteroid families are groups of asteroids that are the product of collisions or of the rotational fission of a parent object. These groups are mainly identified in proper elements or frequencies domains. Because of robotic telescope surveys, the number of known asteroids has increased from $\simeq 10000$ in the early 1990s to more than 750000 nowadays. Traditional approaches for identifying new members of asteroid families, like the hierarchical clustering method (HCM), may struggle to keep up with the growing rate of new discoveries. Here we used machine learning classification algorithms to identify new family members based on the orbital distribution in proper $(a, e, \sin (i))$ of previously known family constituents. We compared the outcome of nine classification algorithms from stand-alone and ensemble approaches. The extremely randomized trees (ExtraTree) method had the highest precision, enabling to retrieve up to 97 per cent of family members identified with standard HCM.
\end{abstract}

Key words: software: data analysis - celestial mechanics - minor planets, asteroids: general.

\section{INTRODUCTION}

Asteroid families are groups of asteroids that form because of collisions or the rotational failure of a parent body. The most widely used method for identifying these groups is the hierarchical clustering method $(\mathrm{HCM})$ in the $(a, e, \sin (i))$ proper element domain, where $a$ is the proper semimajor axis, $e$ is the proper eccentricity, and $i$ the proper inclination. In this method, asteroids are linked to a parent body if their distance in proper element domains, defined through a metric, is less than a critical value, called cutoff. If an asteroid is closer to the parent body than this distance, it is added to the family list. The procedure is then repeated with this asteroid as a new parent body, until no new family members are identified.

This approach was introduced in the early 1990s of the last century when the number of asteroids with proper elements was of the order of $\simeq 10000$ (Zappalá et al. 1990, 1995). Thanks to robotic surveys such as Spacewatch and LINEAR, the number of asteroids for which reliable proper elements are available is nowadays of the order of 750000 , and this number is continually increasing (see DeMeo et al. 2015 for an in-depth discussion of the recent rates of asteroid discoveries). In high-number-density regions of the main belt, standard application of HCM may not be directly applicable: Asteroid families close in proper element spaces may overlap and no longer be recognizable as an individual entity. While alternative implementations of HCM have been proposed to solve this problem (Milani et al. 2014), these approaches may be computationally

\footnotetext{
^E-mail: valerio.carruba@unesp.br
}

expensive and require to obtain solutions for all families in the orbital region where the group resides.

In the last years, several machine learning algorithms have been introduced in the PYTHON programming language and are freely available for solving classification problems. Given a preexisting population, these methods may use the known data to predict if new data belongs, or not, to a given group. In this work, we will investigate if these algorithms can be applied for the purpose of automatically identifying new possible members of a given asteroid family, without the need of obtaining a solution for all the other families in the region. We will then verify how the newly identified asteroids compare to those identified by traditional HCM, and we will introduce parameters to quantify the efficiency of the machine learning algorithms. By studying several algorithms that apply stand-alone or ensemble methods, we aim to identify the method that could perform best for the problem at hand. We will start our work by selecting the asteroid families that are most suited for our analysis, in the next section.

\section{MACHINE LEARNING CLASSIFICATION OF NEW ASTEROID FAMILIES MEMBERS}

In this section, we are going to discuss the implementation of machine learning algorithms for the purpose of classifying new family members. First, we need a consistent way to identify asteroid family members for the training of the algorithms. For this purpose, we turn our attention to the data base of asteroid families available at the Asteroid Families Portal (AFP, http://asteroids.matf.bg.ac.rs/fam/properelements.php: accessed on 2019 December 5; Radović et al. 2017). The web-page-based algorithms allow to automatically obtain asteroid families in a 
data set of 631226 asteroids with synthetic proper elements using standard HCM procedures. New asteroids proper elements, not included in the data base used for the family identification purposes, are also available in this site. For each family available in that data base, with the exception of the very large Flora and Vesta families, and the complicated cases of the Nysa, Polana, and new Polana families, we divided the family sample into two parts, one for objects with absolute magnitude $H<14$, the training sample, and one for the rest, which we call the test sample. The choice of this absolute magnitude value as a cutoff is motivated by the work of Milani et al. (2014), which showed that families computed for asteroids with $H$ $<14$ are not affected by the chaining issue of dynamical families obtained with hierarchical clustering methods. These groups should present minimal or no overlap with other nearby dynamical families.

We required the chosen families to be represented in the Radović et al. (2017) catalogue, to be located in the inner, central, and outer main belt at low inclinations (see Carruba et al. 2013, for a definition of these zones), since in these regions, we found the most numerous families, and also to have at least 10 members with $H<14$ (we include just one case with a lower sample, that of the Massalia family, so as to have a larger number of families located in the inner main belt. This family is most likely the outcome of a cratering event, which explains the lack of bright members; Milani et al. 2019). Twenty-one asteroid families satisfy our selection criteria and will be used for this study. Machine learning methods are applied to the proper $(a, e, \sin (i))$ distribution of the training sample, and used to predict the membership of the test sample population. To check how effective the algorithms are in retrieving asteroid families members, we defined three coefficients. $f_{1}$, or 'Completeness', is the fraction of family members that were retrieved by the machine learning algorithm with respect to the total original population. If, following the notation of Carruba, Aljbaae \& Lucchini (2019), we define as true positive (TPos) asteroids identified as family members by both methods, false positive (FPos) as asteroids identified as family members by the machine learning algorithm alone, and false negative (FNeg) as asteroids not identified as family members only by the machine learning algorithms, it then follows that

$f_{1}=$ Completeness $=\frac{T \text { Pos }}{T P o s+F N e g}=\frac{T P o s}{N_{\mathrm{Or}}}$,

where $N_{\mathrm{Or}}=T P o s+F N e g$ is the number of asteroids in the original family. We can also define a Precision coefficient that yields the ability of the model to avoid predicting false data. This is given by (see equation 3 in Carruba et al. 2019)

Precision $=\frac{T P o s}{T P o s+F P o s}=\frac{T P}{N_{\text {Retr }}}$,

where $N_{\text {Retr }}=$ TPos + FPos is the number of asteroids in the retrieved family. A high value of $f_{1}$ alone indicates that the algorithm may have been successful at retrieving a large part of the original population. But this, alone, is not an indication of a good fit. For instance, imagine having an original small family of $\simeq 100$ members and a retrieved family of $\simeq 10000$ objects, which includes all 100 original members. The Completeness $f_{l}$ coefficient would be 1 in this case. But, clearly, the retrieved family is not a good approximation of the original one, in this case. Conversely, a high value of Precision alone may be associated with a family that is too small. An example of this could be an original family of $\simeq 100$ members and a retrieved family of $\simeq 10$ objects, all true positives. The Precision coefficient would be 100 per cent, but, again, the retrieved family would not be a good representation of the original group. Both coefficients yield useful information, but neither is sufficient alone to identify good retrieved families. As a compromise, and also to use a single parameter instead of two, we introduce the 'final parameter' $(F P)$ as $F P=\frac{1}{\sqrt{2}} \sqrt{(\text { Completeness })^{2}+(\text { Precision })^{2}}$.

In this work, we will apply three classes of machine learning algorithms: stand-alone, bagging, and boosting methods. Standalone methods, as their name suggests, are approaches that use a single algorithm for the classification process. Bagging and boosting methods are ensemble methods that use several stand-alone algorithms. Bootstrap aggregating, often abbreviated as bagging, has each classifier in the ensemble accounted for with an equal weight. An example of bagging methods is the random forest (RF) algorithm, where the outcomes of several random decision trees are combined to achieve very high classification accuracy. Boosting does not account for each classifier with a equal weight, but emphasizes the training cases that previous classifiers misclassified, and the stand-alone classifiers that performed better. Boosting may provide better accuracy than bagging, but it may also be more likely to overfit the training data. More details on the theory behind these methods can be found in Swamynathan (2017).

Each algorithm may depend on one or more free parameters, which need to be optimized. For instance, in the k-Nearest Neighbours $(\mathrm{kNN})$, a free parameter is the number of neighbours to each point. In an ensemble method such as the RF, the number of single stand-alone estimators, or, number of estimators, is another free variable. These parameters, also called hyperparameters, need to be studied on a case by case basis for each given family. In this work, we will use the GridSearchCV approach of the SCIKIT-LEARN package (Pedregosa et al. 2011), which applies the algorithm of interest for a full grid of values of the hyperparameter. We will then apply the best-fitting value found by this approach to each studied family. Mean values of the hyperparameters and of the FP coefficients for the 21 studied asteroid families, with their errors, will be provided for each of the studied algorithms. For families not included in our test sample, a given algorithm can then be applied using the mean value of the hyperparameter found with the analysis of the 21 studied cases. The mean value of the FP coefficient can be used to compare the outcome of different algorithms: the higher its value, the better the algorithm at retrieving family members.

In the next subsection, we will investigate how the selected methods perform the task of classifying new possible members, starting with stand-alone algorithms. All tables referring to results obtained in the next subsections are available in the Appendix.

\subsection{Stand-alone methods}

Here we will investigate the effectiveness of three stand-alone machine learning algorithms for the identification of new family members: linear regression, $\mathrm{kNN}$, and decision tree. We start our analysis with the linear regression method.

\subsubsection{Linear regression algorithm}

Linear regression searches for a linear relationship between an independent variable, $x$ or input, and a dependent one, y or output. For our application, the linear regression algorithm uses the proper element data from the family to train, and then computes a probability for asteroids in the extended data base that they may belong to the family. The asteroid is assumed to be a family member if its computed probability is higher than a threshold value. Since the relationship between the $(a, e, \sin (i))$ proper elements and family membership may be non-linear, we do not expect this algorithm to perform well. Nevertheless, for historical reasons, and since this is 


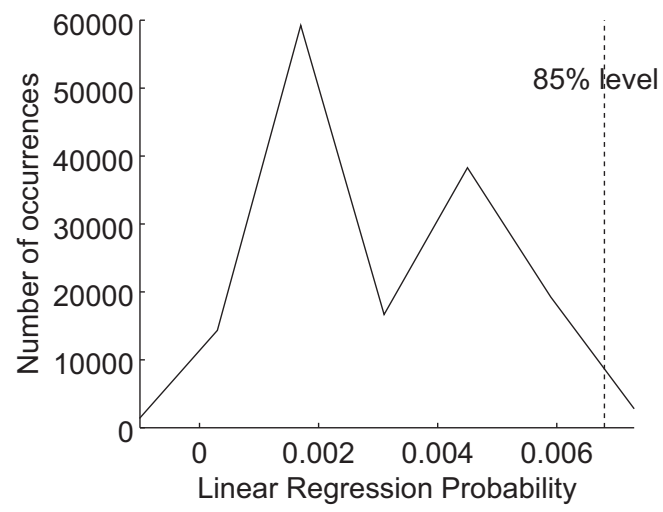

Figure 1. The distribution of probability levels for asteroids in the lowinclination central main belt to belong to the 729 Watsonia family, as obtained by the linear regression algorithm. The vertical dashed line displays a probability value that is higher of those of 85 per cent of the tested population.

one of the most commonly used machine learning algorithms, we will begin our analysis by using linear regression.

Fig. 1 displays a plot of the probability levels of a body to belong to the (729) Watsonia family, for asteroids in the central main belt. On the $y$-axis, we report the number of asteroids in the region that have a given probability of belonging to this family. The value of probabilities changes, but we can define them as a percentage of the maximum probability level. The vertical dashed line in Fig. 1 shows the 85 per cent probability level, which is a probability value that is higher than those computed by the algorithm for 85 per cent of the tested population. Other choices of the probability cutoff are, of course, possible. One has, therefore, to choose the probability level that is most appropriate for a given family. Here we choose the probability level that maximizes values of $F P$, as computed by equation (3). For each family, we tested various values of the probability cutoff, compute the corresponding Completeness, Precision, and FP coefficients, and selected the probability cutoff associated with the highest $F P$. Our results for 21 asteroid families are shown in Table A1. The last column displays the values of the hyperparameter probability cutoff coefficient that satisfy our selection criteria.

For illustrative purposes, we show, for the case of the linear regression algorithm, histograms of hyperparameter value (the lefthand panel of Fig. 2 displays a histogram of the 'probability cutoff' hyperparameter), and of the FP coefficient (right-hand panel of Fig. 2). Similar histograms were generated for the other algorithms studied in this paper, but will not be shown for the sake of brevity. The mean value of the hyperparameter was $50.5 \pm 11.17$, while for $F P$, we obtained $F P=0.71 \pm 0.02$. Since results of this approach, even at the optimal value of the hyperparameter, all tend to overestimate the family and yield low values of the Precision coefficient, the applications of this method may be limited.

\subsubsection{KNN algorithm}

The basic idea is to predict the status of a data point, which, in our context, means if a given asteroid is a member of the family or not, by looking at the $k$ closest neighbours, and then decide by taking a majority vote. For instance, imagine that an asteroid has five neighbours, three of which are members of the family of interest. In this case, since the majority of the neighbours are family members, the asteroid would also be labelled as such. A hyperparameter of this method is the optimal 'number of neighbours' to be considered. The mean value of this parameter for the 21 studied families was $2.35 \pm 1.88$, while for $F P$, we obtained $F P=0.84 \pm 0.08$. Please see Table A2 for a summary of our results.

\subsubsection{Decision tree algorithm}

Decision tree classifications algorithms were first introduced by Quinlan (1986). The concept behind this method is relatively simple: decisions are taken in forms of a tree. Imagine that you want to know the weather of a day in the past. At the top level, we may ask whether the day was sunny or rainy. At a second level, we could ask if the temperature was hot, defined as higher than a threshold, or cold. We could then ask the data if the day was dry or humid, etc. At the final level, the leaf node, the last leaf will classify the data into various categories, like a sunny, warm, dry day, etc. The number of decision nodes to be used in the evaluation of the data, or 'max depth', in the implementation of the decision tree algorithm by SCIKIT-LEARN, is a hyperparameter of this method. For the studied families, the mean value of this parameter was $7.25 \pm 2.91$, while for $F P$, we obtained $F P=0.84 \pm 0.07$. Table A3 reports our results.

\subsection{Bagging classifiers}

In this subsection, we will investigate three cases of bootstrap aggregations: the bagging classifier, the RF, and the extremely randomized trees (ExtraTree).

\subsubsection{Bagging classifier}

This method, also known as bootstrap aggregation, was first introduced by Leo Breiman in 1994. In this approach, the training data is first used to create multiple samples, called the bootstrap samples. Typically, the bootstrap sample size is the same as the original training sample size. However, it contains $3 / 4$ of the original values and randomly chosen replacements for the remaining $1 / 4$, which may lead to repetition of data. Each of the bootstrap samples is used to train an independent classifier, which can be a linear regression or a decision tree algorithm. For regression problems, where the predicted variable is a continuous one, the final model is built based on the average of the predictions of all stand-alone classifiers. For classification problems, like the one at hand in this work, where two outcomes are possible (the asteroid is a member of a family, or it is not), the criterion used for determining the final outcome is a majority vote among the stand-alone classifiers.

Here we use the bagging classifier method using decision trees as stand-alone models. The 'number of neighbours' hyperparameter for the decision trees algorithms is the optimal value found in section (2.1.3). A hyperparameter of this approach is the "number of estimators', which is the number of stand-alone algorithms used by the bagging classifier. Here we investigate the values of these hyperparameter in the range from 1 to 150 . The mean value of the 'number of estimators' was $15.55_{-15.55}^{+18.16}$, while for $F P$, we obtained $F P=0.81 \pm 0.09$. Results for the 21 studied families are displayed in Table A4.

\subsubsection{RF algorithms}

$\mathrm{RF}$ algorithms are an application of bagging classifier methods, with improvements for what concerns the procedure by which the stand-alone method, which is the decision tree, selects the 

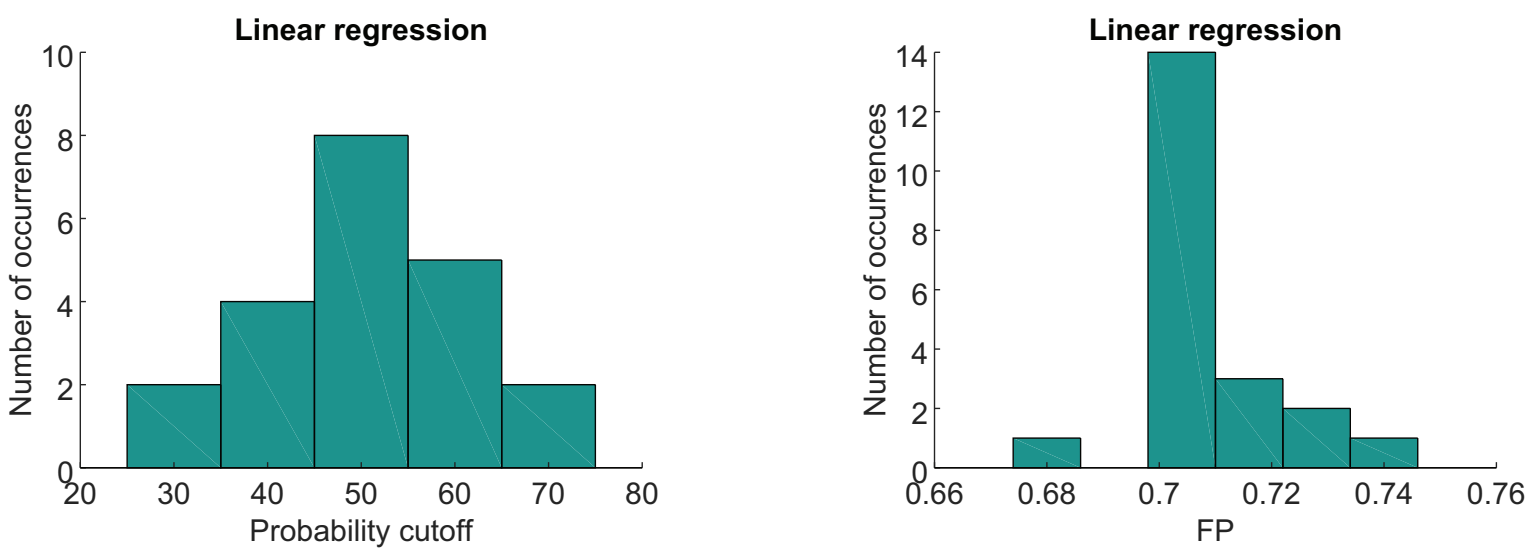

Figure 2. Histograms of the hyperparameter probability cutoff (left-hand panel) and FP coefficient (right-hand panel) for the 21 families studied with the linear regression algorithm.

optimal split point for the various nodes. In normal applications of bagging classifiers, the decision tree algorithms are allowed to look at all variable values in order to produce the optimal split point for the tree structure. As a consequence, the decision trees can have a lot of similarities among them, and their predictions may be somewhat correlated. In RF algorithms, the learning algorithm will only test random samples of variable values. Classification problems are handled by RF algorithms in the same way as bagging classifiers, i.e. by a majority vote of the predictions of each standalone method. A hyperparameter of this method is also the 'number of estimators', which is the number of single decision trees used by the ensemble approach. As in previous sections, we used the GridSearchCV approach to find the best values of this parameter in a range from 1 to 150 . Our results are shown in Table A5. The mean value of the 'number of estimators' hyperparameter was $40.7 \pm 9.56$, while for $F P$, we obtained $F P=0.77 \pm 0.08$.

\subsubsection{Extremely randomized trees (ExtraTree)}

As with the two previous algorithms, this is also a bagging method in which several predictions from different decision trees are combined to obtain a better final result. With respect to the two previous methods, however, the ExtraTree does not use bootstraps, which means that it samples data without replacements. The procedure for creating the tree structure is similar to that of the RF algorithm (see discussion in Section 2.2.2). As for other bagging ensemble methods, a hyperparameter of this algorithm is the "number of estimators'. Again, we used the GridSearchCV approach to find the best value of this parameter in a range from 1 to 150 . Our results are provided in Table A6. The mean value of the 'number of estimators' hyperparameter was $40.95 \pm 29.21$, while for $F P$, we obtained $F P=$ $0.84 \pm 0.10$.

\subsection{Boosting}

Three of the most commonly used boosting algorithms, adaptive boosting (AdaBoost), gradient boosting (Gboost), and the eXtreme gradient boosting (XGboost) will be used in the following subsections.

\subsubsection{AdaBoost algorithm}

Boosting methods are, like bagging ones, ensemble methods where the predictions of several stand-alone approaches are combined to offer a better outcome. With respect to bagging methods, boosting algorithms track the classifiers that provided the least accurate prediction and assign to them lower weights. The final outcome is reached as a weighted average of the outcomes of each standalone classifier. The method used for AdaBoost follows these steps; interested readers can found further details in Swamynathan (2017, chapter 4). At first, all data points and model receive equal weights. The classifiers are trained over the data points, and the data points that were wrongly classified receive a higher weight, so that in the next iteration, they will influence the outcome of the model more than before. Classifiers that had a higher accuracy receive higher weights. The process is iterated until the training data are fitted without significant errors, or the maximum number of estimators is reached. As discussed, the final outcome is achieved by means of weighted averages. An important hyperparameter of this method is the 'number of estimators', which has been tuned with GridSearchCV to find the optimal value in a range from 1 to 150. The mean value of this hyperparameter was $41.25_{-41.25}^{+45.41}$, while for $F P$, we obtained $F P=0.85 \pm 0.06$. Results of the applications of this method are shown in Table A7.

\subsubsection{Gboost algorithm}

While the AdaBoost algorithm used weights to identify weak stand-alone algorithms, Gboost uses gradients. This works in the following way: imagine that one of the stand-alone classifiers used underperforms when compared to others and needs to be corrected by adding a new estimator. At the next iteration of the process, the new estimator will try to fit the residual difference between the data and the predictions of the former weak learner. The process is then repeated until no further improvements are possible. More detailed information on this approach can be found in Swamynathan (2017, chapter 4). As in Section 2.3.1, we use GridSearchCV to find the optimal value of the hyperparameter 'number of estimators', which is $91.45 \pm 52.67$. The mean value of $F P$ was $F P=0.76 \pm 19$. Results are displayed in Table A8.

\subsubsection{XGboost algorithm}

Both the XGboost and the Gboost methods follow the principle of Gboost. XGboost, however, uses a more regularized model, and has performance enhancements, like better support for multicore processing, which permits for faster training times. An in-depth discussion of the theory behind this method would require much 
Table 1. Summary of the best results of the studied machine learning algorithms.

\begin{tabular}{lccccc}
\hline Family & $\begin{array}{c}\text { Members with } \\
H<14\end{array}$ & $f_{1}$ & Prec & $\begin{array}{c}\text { Best } \\
F P\end{array}$ & $\begin{array}{c}\text { Best } \\
\text { estimator }\end{array}$ \\
\hline 20 Massalia & 4 & 0.35 & 0.83 & 0.64 & ExtraTree \\
163 Erigone & 16 & 0.61 & 0.92 & 0.78 & AdaBoost \\
15 Eunomia & 1072 & 0.93 & 0.86 & 0.89 & ExtraTree \\
170 Maria & 392 & 0.83 & 0.87 & 0.85 & Bagging classifier \\
668 Dora & 128 & 0.94 & 0.98 & 0.96 & ExtraTree \\
847 Agnia & 98 & 0.76 & 0.95 & 0.86 & ExtraTree \\
363 Padua & 39 & 0.81 & 0.68 & 0.74 & KNN \\
1726 Hoffmeister & 42 & 0.86 & 0.94 & 0.89 & KNN \\
410 Chloris & 45 & 0.80 & 0.66 & 0.87 & XGBoost \\
808 Merxia & 28 & 0.85 & 0.95 & 0.90 & Decision tree \\
128 Nemesis & 19 & 0.70 & 0.92 & 0.81 & XGBoost \\
569 Misa & 11 & 0.60 & 0.98 & 0.81 & AdaBoost \\
221 Eos & 2043 & 0.88 & 0.90 & 0.89 & ExtraTree \\
24 Themis & 1029 & 0.95 & 0.94 & 0.95 & ExtraTree \\
158 Koronis & 790 & 0.97 & 0.95 & 0.96 & ExtraTree \\
10 Hygiea & 453 & 0.90 & 0.95 & 0.93 & XGBoost \\
375 Ursula & 285 & 0.80 & 0.89 & 0.85 & ExtraTree \\
1040 Klumpkea & 195 & 0.89 & 0.94 & 0.91 & ExtraTree \\
283 Emma & 37 & 0.89 & 0.89 & 0.89 & KNN \\
845 Naema & 18 & 0.92 & 0.95 & 0.94 & ExtraTree \\
490 Veritas & 133 & 0.96 & 0.91 & 0.94 & KNN \\
\hline
\end{tabular}

Note. We report the family identification, the number of members with $H<14$, the values of the 'Completeness' (or $f_{1}$ ), and 'Precision' coefficients associated to the best value of the FP coefficient obtained in our study (displayed in the fifth column), and the name of the estimator that outperformed the other algorithms.

more space, and it is beyond the purposes of this paper. More information can be found in Swamynathan (2017, chapter 4), and references therein. XGBoost depends on a series of hyperparameters. Among them, here we will work with the following parameters, whose definitions are direct quotes from Swamynathan (2017):

(i) eta: the learning rate; default value is 0.3 .

(ii) max_depth: maximum depth of trees; default is 6 .

(iii) colsample_bytree: the fraction of columns to be randomly sampled for each tree; default value is 1 .

(iv) Subsample: the fraction of observations to be randomly sampled for each tree algorithm; default is 1.

(v) alpha: L1 regularization term on weight; default is 1 .

(vi) lambda: L2 regularization term on weights; default value of 1 .

To optimize the values of these parameters, we used the GridSearch CV approach of the SCIKIT-LEARN package. For each given value of a hyperparameter, the method fits the data and search for the most optimal values amongst the studied ones. Here we use the following ranges for our hyperparameters: eta: [0.001, 0.01, 0.1], max_depth: $[2,5,10,20]$, colsample_bytree: $[0.1,0.5,0.8,1]$, Subsample: [0.1,0.5,1], alpha: [0.1,0.5,1], and lambda: [0.1,0.5,1]. As for other ensemble methods, we also tuned the "number of estimators' hyperparameter, and we tested values in a range from 1 to 150 .

Results are shown in Table A9. Values of the eta and Subsample parameters were equal to 0.1 for all cases. The mean values of the other four parameters and of their errors, estimated to be equal to the standard deviations of the distributions, were max_depth $=$ $5 \pm 5$, colsample_bytree $=1.0 \pm 0.1$, alpha $=0.1 \pm 0.1$, and lambda $=0.5 \pm 0.4$, respectively. The mean value of the "number of estimators' hyperparameter was $88.15 \pm 46.71$, while for $F P$, we obtained $F P=0.83 \pm 0.09$.

\section{THE BIG PICTURE}

Each asteroid family is unique in terms of its population, of the effects of the local dynamical environment, its age, its possible interaction with massive asteroids or dwarf planets, etc. It is, therefore, not surprising that the outcome of the studied classification algorithms is different for each of the 21 studied cases. Overall, an important parameter to consider is the size of the sample used to train the classification algorithm. The larger the sample, the better we would expect the outcome of the prediction to be. For each studied family, we verified which algorithm provided the best performance, measured by the FP parameter. Our results are shown in Table 1.

Overall, ExtraTree had the best performance for 10 out of the 21 studied families, followed by KNN with four cases, XGBoost (3 families), AdaBoost ( 2 families), and the bagging classifier and decision tree algorithms, both with one case. ExtraTree performed better with families with a large $N(H<14)$ population, and comes as second best in many other cases (4). KNN appears to perform well for small- or medium-sized families, with less than $\simeq 150$ members. XGBoost is a third possible good choice among the studied algorithms.

Fig. 3 displays an $(a, \sin (i))$ projection of the best results for family membership of the ExtraTree algorithm (Koronis family, panel a), and KNN method (Veritas family, panel b). Black dots display the members of the family, as obtained by the $A F P$, while red circles show the orbital locations of the family members predicted by the machine learning algorithm. Values of $F P$ are higher than 94 per cent for both families, with an excellent performance of the two methods.

To check if the two algorithms that produced the best results also perform well with other families, we studied the case of five asteroid families in the Cybele and highly inclined main belt, which have a statistically significant population of objects with $H<14$ : the families of (480) Hansa, (945) Barcelona, (31) Euphrosyne, (702) Alauda, and (87) Sylvia. We use the optimal values of the 

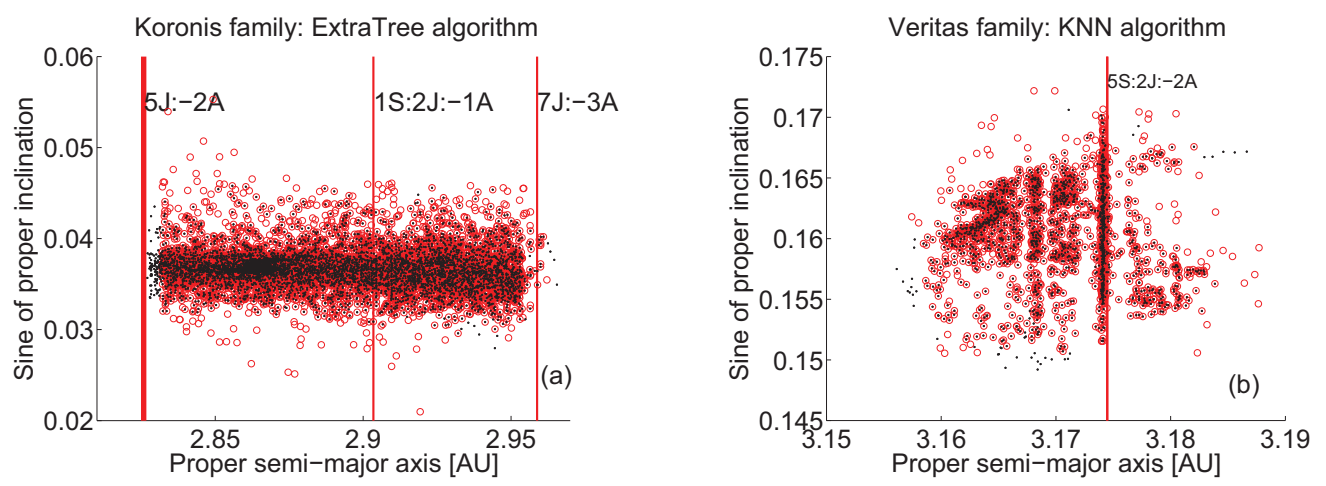

Figure 3. An $(a, \sin (i))$ projection of members of the sampled (black dots) and predicted (red circles) Koronis (panel a) and Veritas (panel (b) asteroid families. Members of the Koronis family were predicted using the ExtraTree algorithm, while members of the Veritas family were determined with the KNN method. Vertical lines display the location of local two- and three-body mean-motion resonances.

Table 2. Results of KNN and ExtraTree for five highly inclined $(\sin (i)>$ $0.3)$ asteroid families.

\begin{tabular}{lccc}
\hline $\begin{array}{l}\text { Family } \\
\text { ID }\end{array}$ & $\begin{array}{c}\text { Members with } \\
H<14\end{array}$ & $\begin{array}{c}\text { KNN } \\
F P\end{array}$ & $\begin{array}{c}\text { ExtraTree } \\
F P\end{array}$ \\
\hline 480 Hansa & 347 & 0.89 & 0.90 \\
945 Barcelona & 88 & 0.84 & 0.88 \\
31 Euphrosyne & 163 & 0.91 & 0.95 \\
702 Alauda & 459 & 0.81 & 0.86 \\
87 Sylvia & 32 & 0.72 & 0.74 \\
\hline
\end{tabular}

hyperparameters found in our previous analysis, which is 2 for the number of neighbours in $\mathrm{KNN}$ and 41 for the number of estimators in the ExtraTree algorithm. Our results are shown in Table 2.

ExtraTree had the best performance in all cases, with values of FP consistently higher than 74 per cent. Based on this analysis, we believe that this algorithm may generally be the best tool to use amongst the studied methods.

\section{UPDATING THE MEMBERSHIP OF ASTEROID FAMILIES}

The main goal of a classification algorithm should be to find new family members. Having established what is the optimal tool, we now try to use it to update the list of family members. We modify the approach and divide the sample of proper elements in a given region into two parts. The first part will go up to the highest numbered member in a given asteroid family. Asteroids in this set of proper elements will be labelled with 1 if they belong to a given family, and 0 otherwise. Machine learning algorithms will then use this set of elements to train so as to predict which asteroids in the remaining set of asteroids with identifications larger than that of the highest numbered object in the family are most likely to be classified as new family members.

We applied this new approach to four asteroid families: (694) Ekard (105 members), (480) Hansa (1484 members), (15) Eunomia (6076 members), and (832) Karin (480 members). These are a small, medium, and large groups in terms of numbers of members. Karin is a subfamily of the larger Koronis group and was included to test how the algorithm performs for subfamilies located in high-number density regions. Contrary to the tests that we run before, we quantify the quality of the fit by checking how the retrieved family compares with respect to the known family in terms of the dispersions of proper elements, since, in principle, we have no information on the family obtainable by standard HCM in the extended sample. In particular, we define an index $d_{\text {std }}$ :

$d_{\text {std }}=\frac{1}{\sqrt{3}} \sqrt{\left(\frac{\sigma\left(a_{\text {retrieved }}\right)}{\sigma\left(a_{\text {known }}\right)}\right)^{2}+\left(\frac{\sigma\left(e_{\text {retrieved }}\right)}{\sigma\left(e_{\text {known }}\right)}\right)^{2}+\left(\frac{\sigma\left(\sin (i)_{\text {retrieved }}\right)}{\sigma\left(\sin (i)_{\text {known }}\right)}\right)^{2}}$,

where $\sigma$ is associated with the standard deviation of the distribution of $(a, e, \sin (i))$ proper elements, and the retrieved and known subscripts refer to the extended family obtained with the algorithm and the known family used for the fitting process. A $d_{\text {std }}$ close to 1 implies that the distribution in proper elements of members of the extended family is compatible with the distribution of the retrieved family, and that results of the machine learning algorithm may be reasonable.

The first three panels of Fig. 4 display $(a, \sin (i))$ projections of three studied families, while the fourth shows a $(a, e)$ section of the fourth, the Karin subfamily of the Koronis group. Black dots identify the original population, while red circles show the new possible family members identified by the ExtraTree algorithm. The scatter in the third dimension for these groups is similar to that observed in the two-dimensional orbital sections. While the distribution of proper elements is tighter in the proper $(a, \sin (i))$ plane for the Ekard, Hansa, Eunomia, and, generally speaking, for most asteroid families, for the very young Karin family, the distribution in proper $(a, e)$ still mostly reflects the dispersion caused by the initial ejection velocity field, which is why, for this family, we decided to plot proper elements in this domain.

We identify 37 new members for the Ekard family, 694 for Hansa, 1550 for Eunomia, and 324 for the Karin group. In all cases, the orbital distribution of the new members is quite compatible with that of the old ones: overall, the mean value of $d_{\text {std }}$ was of $0.97 \pm 0.04$, where the error is defined as the standard deviation of $d_{\text {std }}$. Results are similar for other asteroid families, and the algorithm is able to accurately identify even members of subfamilies located in dense regions of the main belt, such as Karin. Overall, ExtraTree appears to be a reliable tool for automatically detecting new possible family members.

\section{CONCLUSIONS}

In this work, we investigated the possibility of using machine learning classification algorithms for identifying new asteroid families members. We selected 21 asteroid families that, with the exception of the family of (20) Massalia, have a statistically significant population of objects with $H<14$, which is less likely to be affected by the chaining issues that affect standard HCM. We then 


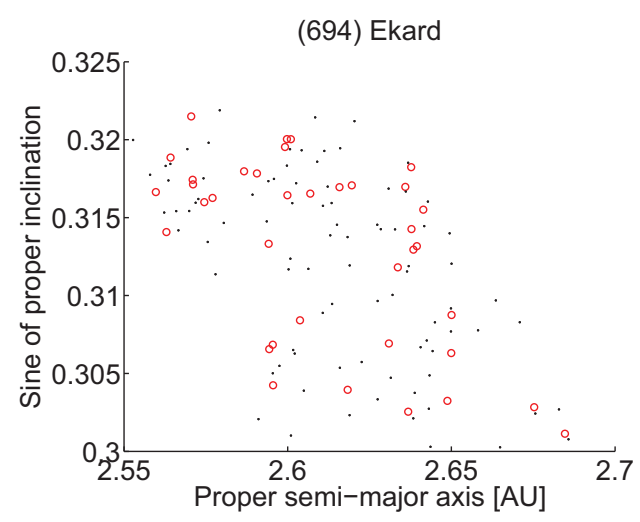

(15) Eunomia

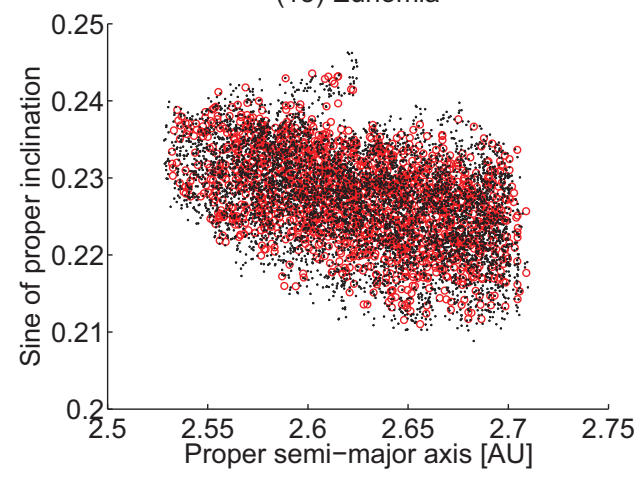

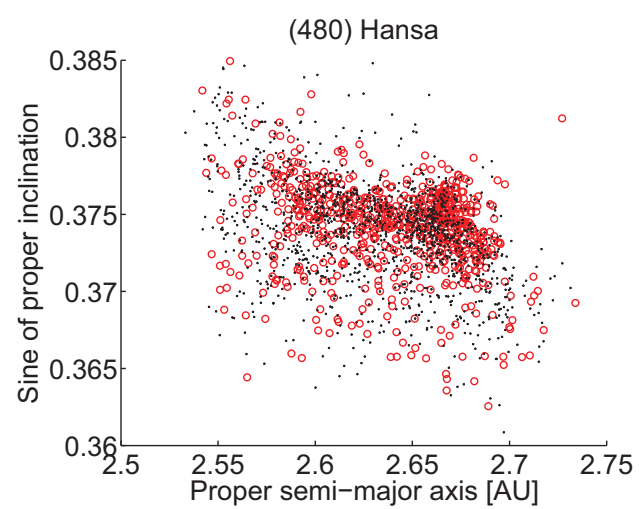

(832) Karin

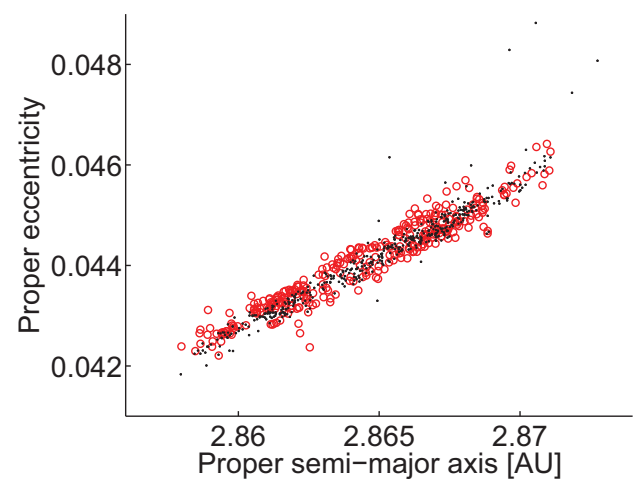

Figure 4. Proper $(a, \sin (i))$ projections of known members (black dots) and retrieved members (red circles) of the Ekard, Hansa, and Eunomia asteroid families. The fourth panel displays an $(a, e)$ projection of members of the known and retrieved Karin subfamily.

applied nine different machine learning algorithms and used three parameters to characterize the efficiency with which the algorithms identify new family members when compared to the results of traditional HCM. We used stand-alone, bagging classifiers, and boosting methods for this purpose. Among the studied algorithms, the ExtraTree had the best performance, followed by the KNN standalone approach and by the XGBoost method. An application to highly inclined asteroid families confirmed that ExtraTree appears to be the most efficient method for this particular classification task.

We adapted the codes for the purpose of automatically updating family memberships, and we tested them with the cases of small, medium-sized, and large asteroid families. In all tested cases, the orbital distribution of the newly found members is highly consistent with the distribution of previously known objects. The PYTHON codes used for this task are publicly available at the GitHub repository (https://github.com/valeriocarruba/Machinelearning-classification-of-new-asteroid-families-members; information on how to download and use the codes is available in the local read-me files). We welcome inputs from the scientific community on how to further improve the performance of these algorithms.

\section{ACKNOWLEDGEMENTS}

We are grateful to an anonymous reviewer for comments and suggestions that helped improve this paper. We would like to thank the São Paulo State Science Foundation (FAPESP, grant 2018/20999-6), the Brazilian National Research Council (CNPq, grant 301577/2017-0, 310317/20169), and the Coordination for the Improvement of Higher
Education Personnel (CAPES, grant 88887.374148/201900). We acknowledge the use of data from the Asteroid Dynamics Site (AstDys) (http://hamilton.dm.unipi.it/astdys, Knežević; Milani 2003) and the Asteroid Families Portal (AFP, http://asteroids.matf.bg.ac.rs/fam/properelements.php; Radović et al. 2017). We also acknowledge the use of exercises proposed by machine learning courses at DataCamp (www.datacamp.com), which either inspired or helped in the development of the software used in this work. Finally, we are grateful to Edmilson Roma de Oliveira for discussions that motivated this work.

\section{REFERENCES}

Carruba V., Domingos R. C., Nesvorný D., Roig F., Huaman M. E., Souami D., 2013, MNRAS, 433, 2075

Carruba V., Aljbaae S., Lucchini A., 2019, MNRAS, 488, 1377

DeMeo F. E., Alexander C. M. O’D., Walsh K. J., Chapman C. R., Binzel

R. P., 2015, Asteroids IV. Univ. Arizona Press, Tucson, AZ

Knežević Z., Milani A., 2003, A\&A, 403, 1165

Milani A., Cellino A., Knežević Z., Novaković B., Spoto F., Paolicchi P., 2014, Icarus, 239, 46

Milani A., Knežević Z., Spoto F., Paolicchi P., 2019, A\&A, 622, A47

Pedregosa F . et al., 2011, J. Mach. Learn. Res., 12, 2825

Quinlan J. R., 1986, Mach. Learn., 1, 81

Radović V., Novaković B., Carruba V., Marceta D., 2017, MNRAS, 470, 576

Swamynathan M., 2017, Mastering Machine Learning with Python in Six Steps, Apress, New York, NY

Zappalá V., Cellino A., Farinella P., Knežević Z., 1990, AJ, 100, 2030

Zappalá V., Bendjoya Ph., Cellino A., Farinella P., Froeschlé C., 1995, Icarus, 116, 291 


\section{APPENDIX A:}

In this appendix, we report the results of the application of nine machine learning algorithms to 21 asteroid families. See Section 2 for the descriptions of the algorithms and of the listed parameters.

Table A1. The table reports the asteroid families from the Asteroid Families Portal, the number of their members, the number of members with $H<14$, the optimal values of 'Completeness' $\left(f_{1}\right)$, 'Precision' $($ Prec $)$, and 'final parameter' FP coefficients, and the corresponding value of the hyperparameter probability cutoff, for the families studied with the linear regression algorithm.

\begin{tabular}{lcccccc}
\hline Family & $\begin{array}{c}\text { Number of } \\
\text { ID }\end{array}$ & $\begin{array}{c}\text { Number of } \\
\text { members } H<14\end{array}$ & $f_{1}$ & Prec & $F P$ & Cutoff \\
\hline 20 Massalia & 6400 & 4 & 1.00 & 0.14 & 0.71 & 50.0 \\
163 Erigone & 2979 & 16 & 0.96 & 0.09 & 0.68 & 30.0 \\
15 Eunomia & 6076 & 1072 & 1.00 & 0.10 & 0.72 & 40.0 \\
170 Maria & 4183 & 392 & 1.00 & 0.12 & 0.71 & 40.0 \\
668 Dora & 1677 & 128 & 1.00 & 0.04 & 0.71 & 50.0 \\
847 Agnia & 4432 & 98 & 1.00 & 0.12 & 0.71 & 60.0 \\
363 Padua & 807 & 39 & 1.00 & 0.02 & 0.71 & 60.0 \\
1726 Hoffmeister & 2396 & 42 & 0.99 & 0.09 & 0.70 & 60.0 \\
410 Chloris & 449 & 45 & 1.00 & 0.01 & 0.71 & 30.0 \\
808 Merxia & 1624 & 28 & 1.00 & 0.03 & 0.71 & 50.0 \\
128 Nemesis & 1449 & 19 & 1.00 & 0.02 & 0.71 & 50.0 \\
569 Misa & 723 & 11 & 1.00 & 0.03 & 0.71 & 60.0 \\
221 Eos & 14661 & 2043 & 1.00 & 0.19 & 0.72 & 40.0 \\
24 Themis & 5946 & 1029 & 1.00 & 0.29 & 0.74 & 50.0 \\
158 Koronis & 7294 & 790 & 0.93 & 0.47 & 0.73 & 60.0 \\
10 Hygiea & 6224 & 453 & 1.00 & 0.12 & 0.71 & 40.0 \\
375 Ursula & 2214 & 195 & 0.99 & 0.21 & 0.72 & 70.0 \\
1040 Klumpkea & 2840 & 37 & 1.00 & 0.25 & 0.73 & 50.0 \\
283 Emma & 610 & 18 & 1.00 & 0.01 & 0.71 & 50.0 \\
845 Naema & 418 & 133 & 0.99 & 0.11 & 0.71 & 70.0 \\
49 Veritas & 1805 & & & & 50.71 & 50.0 \\
\hline
\end{tabular}

Table A2. The table reports the asteroid families from the Asteroid Families Portal, the optimal value of the 'number of neighbours' hyperparameter used for each of the studied families, and the values of the $f_{1}$, Prec, and FP coefficients for families identified with the KNN algorithm.

\begin{tabular}{lcccc}
\hline Family & $\begin{array}{c}\text { Number of } \\
\text { neighbours }\end{array}$ & $f_{1}$ & Prec & $F P$ \\
\hline 20 Massalia & 1 & 0.39 & 0.80 & 0.63 \\
163 Erigone & 1 & 0.70 & 0.76 & 0.73 \\
15 Eunomia & 4 & 0.89 & 0.85 & 0.87 \\
170 Maria & 4 & 0.81 & 0.85 & 0.83 \\
668 Dora & 2 & 0.93 & 0.96 & 0.95 \\
847 Agnia & 1 & 0.87 & 0.82 & 0.85 \\
363 Padua & 1 & 0.81 & 0.68 & 0.74 \\
1726 Hoffmeister & 1 & 0.86 & 0.94 & 0.89 \\
410 Chloris & 2 & 0.77 & 0.67 & 0.72 \\
808 Merxia & 4 & 0.56 & 0.91 & 0.75 \\
128 Nemesis & 2 & 0.57 & 0.92 & 0.76 \\
569 Misa & 1 & 0.68 & 0.74 & 0.71 \\
221 Eos & 1 & 0.89 & 0.85 & 0.87 \\
24 Themis & 1 & 0.94 & 0.91 & 0.93 \\
158 Koronis & 1 & 0.96 & 0.92 & 0.94 \\
10 Hygiea & 2 & 0.80 & 0.95 & 0.88 \\
375 Ursula & 1 & 0.84 & 0.80 & 0.82 \\
1040 Klumpkea & 8 & 0.91 & 0.90 & 0.90 \\
283 Emma & 6 & 0.89 & 0.89 & 0.89 \\
845 Naema & 2 & 0.99 & 0.81 & 0.91 \\
490 Veritas & & 0.96 & 0.91 & 0.94 \\
\hline
\end{tabular}

Table A3. The table reports the asteroid families from the Asteroid Families Portal, the optimal value of the 'Max Depth' hyperparameter used for each of the studied families, and the values of the $f_{1}$, Prec, and FP coefficients for families identified with the decision tree algorithm.

\begin{tabular}{lcccc}
\hline Family & $\begin{array}{c}\text { Maximum } \\
\text { ID }\end{array}$ & $f_{1}$ & Prec & $F P$ \\
\hline 20 Massalia & 3 & 0.22 & 0.81 & 0.60 \\
163 Erigone & 7 & 0.63 & 0.89 & 0.77 \\
15 Eunomia & 12 & 0.90 & 0.81 & 0.86 \\
170 Maria & 6 & 0.85 & 0.81 & 0.83 \\
668 Dora & 6 & 0.78 & 0.92 & 0.85 \\
847 Agnia & 6 & 0.86 & 0.80 & 0.83 \\
363 Padua & 4 & 0.78 & 0.68 & 0.73 \\
1726 Hoffmeister & 5 & 0.66 & 0.98 & 0.83 \\
410 Chloris & 6 & 0.77 & 0.65 & 0.71 \\
808 Merxia & 5 & 0.85 & 0.95 & 0.90 \\
128 Nemesis & 5 & 0.73 & 0.89 & 0.81 \\
569 Misa & 4 & 0.73 & 0.62 & 0.68 \\
221 Eos & 14 & 0.87 & 0.84 & 0.85 \\
24 Themis & 7 & 0.94 & 0.91 & 0.93 \\
158 Koronis & 9 & 0.96 & 0.96 & 0.96 \\
10 Hygiea & 9 & 0.89 & 0.92 & 0.91 \\
375 Ursula & 14 & 0.74 & 0.79 & 0.76 \\
1040 Klumpkea & 7 & 0.85 & 0.90 & 0.88 \\
283 Emma & 8 & 0.79 & 0.92 & 0.85 \\
845 Naema & 5 & 0.80 & 0.97 & 0.89 \\
490 Veritas & 6 & 0.87 & 0.94 & 0.91 \\
\hline
\end{tabular}


Table A4. The table reports the asteroid families from the Asteroid Families Portal, the optimal value of the 'number of estimators' hyperparameter used for each of the studied families, and the values of the $f_{1}$, Prec, and FP coefficients for families identified with the 'Bagging classifier' algorithm.

\begin{tabular}{lcccc}
\hline Family & $\begin{array}{c}\text { Number of } \\
\text { estimators }\end{array}$ & $f_{1}$ & Prec & $F P$ \\
\hline 20 Massalia & 1 & 0.01 & 0.67 & 0.47 \\
163 Erigone & 4 & 0.31 & 0.92 & 0.69 \\
15 Eunomia & 32 & 0.90 & 0.74 & 0.82 \\
170 Maria & 19 & 0.83 & 0.87 & 0.85 \\
668 Dora & 19 & 0.72 & 0.94 & 0.84 \\
847 Agnia & 14 & 0.84 & 0.89 & 0.86 \\
363 Padua & 2 & 0.51 & 0.80 & 0.67 \\
1726 Hoffmeister & 5 & 0.53 & 0.94 & 0.76 \\
410 Chloris & 4 & 0.73 & 0.89 & 0.81 \\
808 Merxia & 6 & 0.70 & 0.87 & 0.79 \\
128 Nemesis & 5 & 0.46 & 0.63 & 0.55 \\
569 Misa & 3 & 0.77 & 0.64 & 0.71 \\
221 Eos & 4 & 0.90 & 0.74 & 0.83 \\
24 Themis & 80 & 0.87 & 0.84 & 0.86 \\
158 Koronis & 17 & 0.96 & 0.95 & 0.96 \\
10 Hygiea & 2 & 0.89 & 0.93 & 0.91 \\
375 Ursula & 42 & 0.88 & 0.76 & 0.82 \\
1040 Klumpkea & 3 & 0.88 & 0.92 & 0.90 \\
283 Emma & 14 & 0.70 & 0.95 & 0.84 \\
845 Naema & 14 & 0.57 & 0.96 & 0.79 \\
490 Veritas & 22 & 0.75 & 0.96 & 0.86 \\
\hline
\end{tabular}

Table A5. The table reports the asteroid families from the Asteroid Families Portal, the optimal value of the 'Number of estimators' hyperparameter used for each of the studied families, and the values of the $f_{1}$, Prec, and FP coefficients for families identified with the RF method.

\begin{tabular}{lcccc}
\hline Family & $\begin{array}{c}\text { Number of } \\
\text { ID }\end{array}$ & $f_{1}$ & Prec & $F P$ \\
\hline 20 Massalia & 46 & 0.00 & 0.00 & 0.00 \\
163 Erigone & 37 & 0.07 & 0.97 & 0.69 \\
15 Eunomia & 48 & 0.68 & 0.95 & 0.83 \\
170 Maria & 48 & 0.60 & 0.96 & 0.80 \\
668 Dora & 29 & 0.55 & 1.00 & 0.81 \\
847 Agnia & 47 & 0.56 & 0.99 & 0.81 \\
363 Padua & 46 & 0.22 & 0.85 & 0.62 \\
1726 Hoffmeister & 49 & 0.36 & 0.99 & 0.75 \\
410 Chloris & 46 & 0.43 & 0.94 & 0.73 \\
808 Merxia & 28 & 0.60 & 1.00 & 0.82 \\
128 Nemesis & 35 & 0.23 & 0.94 & 0.69 \\
569 Misa & 45 & 0.10 & 0.92 & 0.66 \\
221 Eos & 40 & 0.61 & 0.97 & 0.81 \\
24 Themis & 46 & 0.82 & 0.98 & 0.90 \\
158 Koronis & 47 & 0.91 & 0.98 & 0.94 \\
10 Hygiea & 49 & 0.67 & 0.99 & 0.85 \\
375 Ursula & 49 & 0.36 & 0.96 & 0.72 \\
1040 Klumpkea & 16 & 0.63 & 0.99 & 0.83 \\
283 Emma & 41 & 0.22 & 0.96 & 0.71 \\
845 Naema & 22 & 0.24 & 1.00 & 0.73 \\
490 Veritas & 46 & 0.58 & 0.98 & 0.81 \\
\hline
\end{tabular}

Table A6. The table reports the asteroid families from the Asteroid Families Portal, the optimal value of the 'Number of estimators' hyperparameter used for each of the studied families, and the values of the $f_{1}$, Prec, and FP coefficients for families identified with the ExtraTree method.

\begin{tabular}{lcccc}
\hline Family & $\begin{array}{c}\text { Number of } \\
\text { estimators }\end{array}$ & $f_{1}$ & Prec & $F P$ \\
\hline 20 Massalia & 1 & 0.35 & 0.83 & 0.64 \\
163 Erigone & 21 & 0.22 & 0.90 & 0.66 \\
15 Eunomia & 80 & 0.93 & 0.86 & 0.89 \\
170 Maria & 9 & 0.80 & 0.86 & 0.83 \\
668 Dora & 85 & 0.94 & 0.98 & 0.96 \\
847 Agnia & 67 & 0.76 & 0.95 & 0.86 \\
363 Padua & 9 & 0.52 & 0.86 & 0.71 \\
1726 Hoffmeister & 25 & 0.62 & 0.99 & 0.83 \\
410 Chloris & 43 & 0.74 & 0.86 & 0.80 \\
808 Merxia & 62 & 0.33 & 0.94 & 0.70 \\
128 Nemesis & 9 & 0.28 & 0.85 & 0.63 \\
569 Misa & 5 & 0.63 & 0.83 & 0.73 \\
221 Eos & 97 & 0.88 & 0.90 & 0.89 \\
24 Themis & 73 & 0.95 & 0.94 & 0.95 \\
158 Koronis & 38 & 0.97 & 0.95 & 0.96 \\
10 Hygiea & 45 & 0.85 & 0.95 & 0.90 \\
375 Ursula & 135 & 0.80 & 0.89 & 0.85 \\
1040 Klumpkea & 104 & 0.89 & 0.94 & 0.91 \\
283 Emma & 65 & 0.75 & 0.96 & 0.86 \\
845 Naema & 5 & 0.92 & 0.95 & 0.94 \\
490 Veritas & 42 & 0.92 & 0.94 & 0.93 \\
\hline
\end{tabular}

Table A7. The table reports the asteroid families from the Asteroid Families Portal, the optimal value of the 'Number of estimators' hyperparameter used for each of the studied families, and the values of the $f_{1}$, Prec, and FP coefficients for families identified with the AdaBoost method.

\begin{tabular}{lcccc}
\hline Family & $\begin{array}{c}\text { Number of } \\
\text { estimators }\end{array}$ & $f_{1}$ & Prec & FP \\
\hline 20 Massalia & 2 & 0.22 & 0.81 & 0.60 \\
163 Erigone & 147 & 0.61 & 0.92 & 0.78 \\
15 Eunomia & 42 & 0.93 & 0.81 & 0.87 \\
170 Maria & 62 & 0.77 & 0.90 & 0.84 \\
668 Dora & 66 & 0.84 & 0.98 & 0.92 \\
847 Agnia & 20 & 0.73 & 0.97 & 0.86 \\
363 Padua & 11 & 0.36 & 0.93 & 0.70 \\
1726 Hoffmeister & 3 & 0.57 & 0.99 & 0.81 \\
410 Chloris & 3 & 0.82 & 0.87 & 0.85 \\
808 Merxia & 3 & 0.61 & 0.96 & 0.80 \\
128 Nemesis & 4 & 0.71 & 0.91 & 0.81 \\
569 Misa & 4 & 0.60 & 0.98 & 0.81 \\
221 Eos & 144 & 0.88 & 0.87 & 0.88 \\
24 Themis & 92 & 0.92 & 0.95 & 0.94 \\
158 Koronis & 8 & 0.96 & 0.96 & 0.96 \\
10 Hygiea & 7 & 0.89 & 0.93 & 0.91 \\
375 Ursula & 50 & 0.72 & 0.84 & 0.78 \\
1040 Klumpkea & 78 & 0.82 & 0.93 & 0.88 \\
283 Emma & 72 & 0.67 & 0.92 & 0.81 \\
845 Naema & 5 & 0.64 & 0.97 & 0.82 \\
490 Veritas & 4 & 0.87 & 0.94 & 0.91 \\
\hline
\end{tabular}


Table A8. The table reports the asteroid families from the Asteroid Families Portal, the optimal value of the 'Number of estimators' hyperparameter used for each of the studied families, and the values of the $f_{1}$, Prec, and $F P$ coefficients for families identified with the gradient boosting method.

\begin{tabular}{lcccc}
\hline Family & $\begin{array}{c}\text { Number of } \\
\text { estimators }\end{array}$ & $f_{1}$ & Prec & FP \\
\hline ID Massalia & 2 & 0.30 & 0.80 & 0.60 \\
163 Erigone & 83 & 0.59 & 0.89 & 0.75 \\
15 Eunomia & 127 & 0.90 & 0.83 & 0.87 \\
170 Maria & 107 & 0.78 & 0.86 & 0.82 \\
668 Dora & 133 & 0.80 & 0.99 & 0.90 \\
847 Agnia & 68 & 0.79 & 0.86 & 0.82 \\
363 Padua & 9 & 0.70 & 0.50 & 0.61 \\
1726 Hoffmeister & 7 & 0.51 & 0.69 & 0.60 \\
410 Chloris & 47 & 0.80 & 0.55 & 0.69 \\
808 Merxia & 42 & 0.70 & 0.74 & 0.72 \\
128 Nemesis & 78 & 0.14 & 0.12 & 0.13 \\
569 Misa & 12 & 0.36 & 0.57 & 0.48 \\
221 Eos & 131 & 0.85 & 0.84 & 0.85 \\
24 Themis & 141 & 0.96 & 0.95 & 0.96 \\
158 Koronis & 133 & 0.96 & 0.95 & 0.96 \\
10 Hygiea & 140 & 0.88 & 0.95 & 0.92 \\
375 Ursula & 135 & 0.79 & 0.83 & 0.81 \\
2840 Klumpkea & 147 & 0.88 & 0.91 & 0.90 \\
845 Naema & 143 & 0.56 & 0.93 & 0.81 \\
490 Veritas & 1 & 0.81 & 0.75 & 0.78 \\
\hline
\end{tabular}

Table A9. The table reports the asteroid families from the Asteroid Families Portal, the optimal value of the 'number of estimators', eta, max_depth, colsample_bytree, Subsample, alpha, and lambda hyperparameters used for each of the studied families, and the values of the $f_{1}$, precision (Prec), and $F P$ coefficients for families identified with the eXtreme gradient boosting (XGBoost) method.

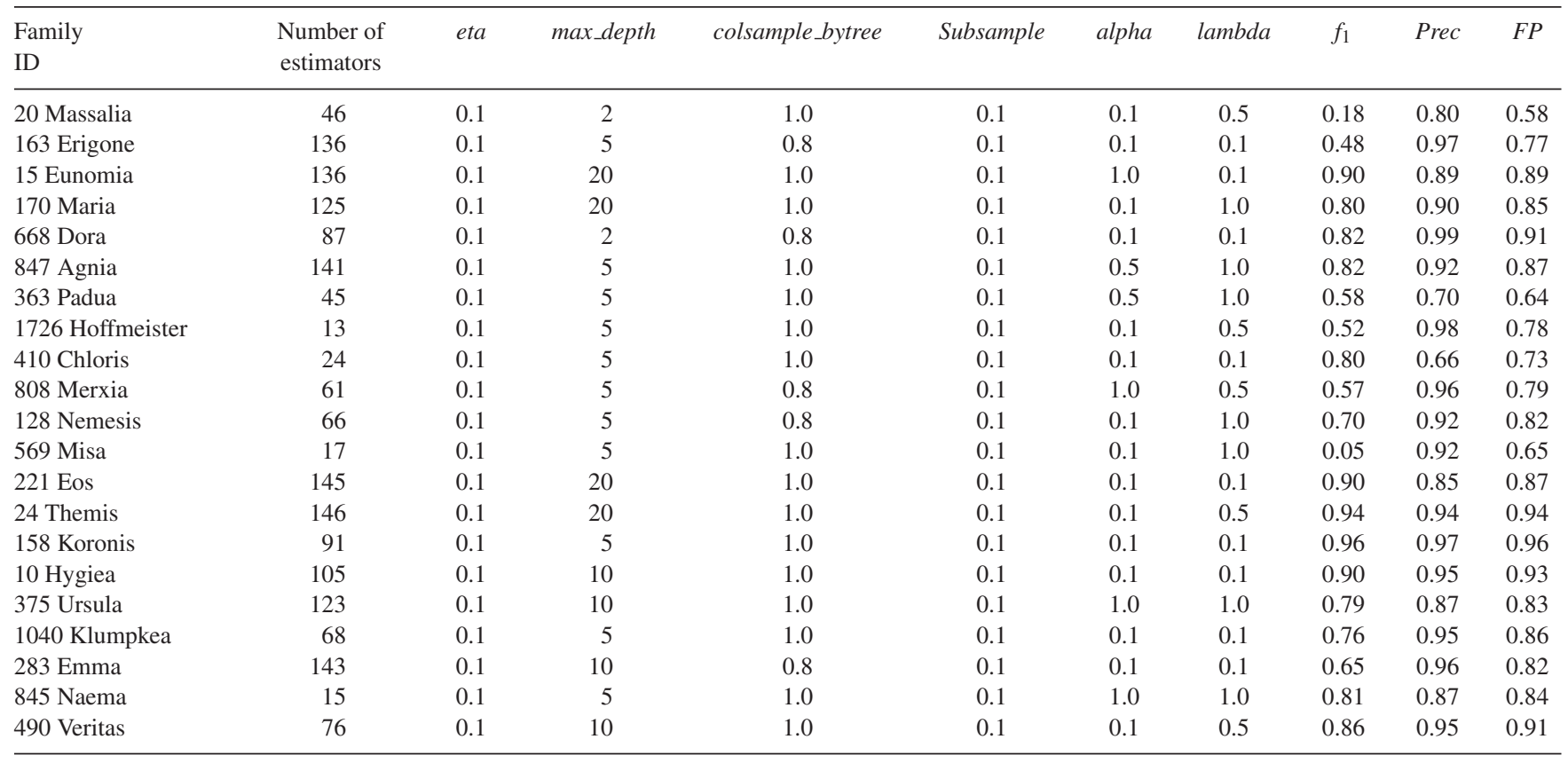

This paper has been typeset from a $\mathrm{T}_{\mathrm{E}} \mathrm{X} / \mathrm{L} \mathrm{A} \mathrm{E} \mathrm{X}$ file prepared by the author. 